\title{
Valor predictivo de la clasificación de Knosp en el grado de resección quirúrgica de los macroadenomas invasivos. Estudio prospectivo de una serie de 23 casos
}

\author{
J. Enseñat; A. Ortega**; T. Topcewski; J. Vilalta; G. Obiols*; J. Mesa* y J. Sahuquillo
}

Servicios de Neurocirugía, Endocrinología* y Anatomía Patológica**. Hospital Universitario Vall d'Hebron. Universidad Autónoma de Barcelona. España.

Resumen

Objetivos. Analizar y valorar el grado de resección y las complicaciones de la cirugía transesfenoidal en una serie de 23 casos de macroadenomas con invasión del seno cavernoso evaluados mediante la clasificación de Knosp.

Material, métodos y resultados. Estudio prospectivo de 22 pacientes (23 operaciones) intervenidos en nuestro centro entre Mayo del 2002 y Diciembre del 2004 de macroadenomas hipofisarios con diferentes grados de invasión del seno cavernoso según la clasificación de Knosp ${ }^{15}$. Entre las variables del estudio se incluyeron los grados de invasión y de resección postoperatoria con un seguimiento radiológico medio a largo plazo de 15 meses.

Nuestra serie consta de 15 varones y 7 mujeres, con una edad media de 48 años (27 - 75 años). Todos ellos presentaban macroadenomas con afectación de uno o ambos senos cavernosos. De acuerdo con la clasificación de Knosp 4 pacientes fueron grado 1, 2 grado 2, 1 grado 3 y 16 grado 4. En 20 casos se utilizó una vía transesfenoidal clásica y en tres casos se hizo un abordaje transesfenoidal endoscópico. Según la RMN postoperatoria los grados de resección fueron: completo o total en todos los pacientes con grados 1 y 2 y en sólo 2 pacientes con grado 4 ; subtotal $(>80 \%)$ en 1 paciente con grado 3 y en 6 pacientes con grado 4 y parcial $(<80 \%)$ en 7 pacientes con grado 4 de Knosp. Se compararon los grados de resección versus los grados de invasión mediante el Test exacto de Fisher y las diferencias no fueron estadísticamente significativas $(\mathrm{p}=\mathbf{0 . 1 2})$.

Discusión y conclusiones. Si bien únicamente la clasificación radiológica de Knosp por si sola no puede predecir el comportamiento biológico del tumor o si la pared medial del seno cavernoso está infiltrada o desplazada, en nuestra serie los tumores de grado 4 han sido los que han presentado un peor resultado de acuerdo al grado de resección. En los tumores que invaden el seno

Recibido; 20-12-05. Aceptado: 19-02-06 cavernoso, incluso en los casos donde la carótida está englobada es posible realizar resecciones completas con una morbimortalidad aceptable.

PALABRAS CLAVE: Macroadenomas invasivos. Seno cavernoso. Abordaje transesfenoidal. Clasificación radiológica.

Predicitve value of the Knosp classification in the staging of invasive pituitary macroadenomas. A Prospective study of 23 cases.

Summary

Objective. To evaluate our experience regarding the treatment of pituitary macroadenomas with cavernous sinus invasion in a series of $\mathbf{2 3}$ cases of transphenoidal resection.

Materials, methods and results. Twenty two patients, fifteen males and seven females, with ages ranging from 27 to 75 (mean of 48), were operated under protocol by a single surgeon between May of 2002 and December of 2004. Preoperatively all lesions were diagnosed by MRI and staged according to the Knosp classification. All tumors had extension to one or both cavernous sinuses. Four patients were considered to be grade 1, two grade 2 , one grade 3 and sixteen grade 4 . Twenty three operations were performed on twenty-two patients. Twenty cases were the standard transsphenoidal approach, and three were endoscopic. Postoperatively, the excision was classified as Complete or Total, Subtotal or Partial. Mean follow up was 15 months. The variables considered for analysis include invasion and resection grades. All six patients with graded 1 and 2 lesions and two patients with grade 4 lesions underwent a complete resection. Subtotal (greater than $\mathbf{8 0} \%$ ) excision was achieved in one patient with a grade 3 tumor and six patients with grade 4 tumors. The remaining seven patients with grade 4 adenomas had a Partial (less than $\mathbf{8 0} \%$ ) excision. We compare de resection grade versus invasion grade with exact Fisher test. And there is not 
estadistical difference $(\mathrm{p}=\mathbf{0 . 1 2})$.

Conclusion. The Knosp classification alone cannot predict the behavior of these tumors. In our experience, despite tumor extension to the cavernous sinus, pituitary macroadenomas can be safely resected with low morbidity and mortality.

KEY WORDS: Invasive macroadenomas. Cavernous sinus. Transsphenoidal approach. Radiologic classification.

\section{Introducción}

Los macroadenomas hipofisarios invasivos representan una forma intermedia entre los adenomas bien delimitados y los carcinomas de hipófisis ${ }^{22}$. A pesar de que estos tumores invaden las estructuras anatómicas contiguas, en especial los senos cavernosos y las estructuras óseas clivales, histopatológicamente son clasificados como benignos $^{21}$. Microscópicamente, la invasión de las estructuras contiguas consiste en células aisladas, grupos celulares o bien en forma de una lengüeta que diseca los diferentes planos conectivos durales ${ }^{22}$. Aunque histológicamente en estos tumores podemos hallar una mayor celularidad, pleomorfismo celular y un índice mitótico elevado, no se puede afirmar que existan diferencias histológicas que nos permitan diferenciarlos y predecir su agresividad y crecimiento $^{22}$.

El tratamiento quirúrgico de los macroadenomas que invaden el seno cavernoso continúa siendo motivo de controversia. Entre un 6 y $10 \%{ }^{1,7}$ de los adenomas de hipófisis crecen comprimiendo las estructuras anatómicas próximas y se extienden hacia el espacio paraselar. Diferentes autores han utilizado diversos criterios para clasificar y valorar la agresividad e invasividad de los macroadenomas. Los marcadores inmunohistoquímicos como el Ki-672,13, la catepsina B o la metaloproteasa-9 (MMP-9) ${ }^{27}$ han dado resultados contradictorios. También se han definido otros factores a nivel genético y molecular que pueden condicionar el grado de crecimiento y la invasividad de los adenomas. Entre estos factores destacan las anomalías de la p $27^{16}$, la disminución en la expresión del gen $\mathrm{nm} 23^{23}$ y la ausencia del gen $\mathrm{p} 53^{24}$.

Aunque las nuevas técnicas de resonancia magnética (RM) permiten una mejor definición de las estructuras anatómicas del espacio paraselar ${ }^{8,11,19}$ continúan siendo poco sensibles para definir con certeza la invasión de éste y en especial del seno cavernoso ${ }^{7,14}$. Los factores anatómicos y estructurales de la pared medial del seno cavernoso parecen jugar un papel fundamental en la invasión tumoral. Una de las grandes controversias en los últimos años, ha sido la definición estructural de dicha pared. Inicialmente se creía que la pared medial del seno cavernoso no existía como tal sino que era la propia cápsula de la glándula hipofisaria la que constituía la pared en sí. Sin embargo, estudios anatómicos recientes han confirmado que existe una pared dural frágil separada de la hipófisis ${ }^{26}$. Otros autores incluso han evidenciado la existencia de defectos estructurales en ésta ${ }^{27}$. Dichos defectos facilitan la invasividad del tumor hacia el interior del seno cavernoso.

Los avances en las técnicas quirúrgicas y la utilización de la endoscopia en el abordaje transesfenoidal han permitido ser cada vez más efectivos en el tratamiento de estos tumores ${ }^{2,6}$. El objetivo de nuestro estudio es evaluar los resultados quirúrgicos en una cohorte de 23 pacientes con macroadenomas invasivos y comprobar el grado de resección postoperatoria en función del grado de invasividad preoperatoria definido por la clasificación de Knosp y su valor predictivo del éxito quirúrgico ${ }^{15}$.

\section{Pacientes y métodos}

En este estudio se incluyeron de forma prospectiva 23 pacientes en el periodo comprendido entre Mayo 2002 y Diciembre del 2004. Todos los pacientes presentaban macroadenomas que invadían el seno cavernoso de forma uni o bilateral. Todos ellos fueron intervenidos mediante un abordaje transesfenoidal. En 20 casos se realizó un abordaje transesfenoidal clásico sublabial y en tres un abordaje transesfenoidal endoscópico. Uno de los pacientes intervenidos por vía endoscópica presentaba restos tumorales de una intervención previa practicada tres meses antes a través de un abordaje sublabial clásico.

\section{Estudio preoperatorio}

Todos los pacientes fueron evaluados según criterios clínicos, radiológicos, y endocrinológicos. Clínicamente se diferenció entre el debut clínico agudo o subagudo (apoplejía hipofisaria) y los pacientes con sintomatología neurológica y/o endocrina más insidiosa. En todos los casos se realizaron campimetrías computarizadas SITA 24-2 para definir el grado de afectación del campo visual. Endocrinológicamente se evaluó la integridad del eje hipotalámo-hipofisario mediante la determinación de las concentraciones plasmáticas de FSH, LH, ACTH, cortisol plasmático, GH, Prolactina, TSH, T4 libre, estradiol (mujeres) y testosterona (hombres).

El estudio neurorradiológico, incluyó en todos los casos un TC helicoidal de las fosas nasales y una RM cerebral de 1.5 T. En la RM se evaluó la invasión del seno cavernoso de acuerdo con la clasificación de Knosp et a ${ }^{15}$. Estos autores proponen la clasificación de los tumores que invaden el seno cavernoso en cinco grados $(0,1,2,3$ y 4$)$ de acuerdo con los siguientes criterios:

Grado 0: el tumor no invade el seno cavernoso. Todas las estructuras anatómicas intracavernosas están preserva- 


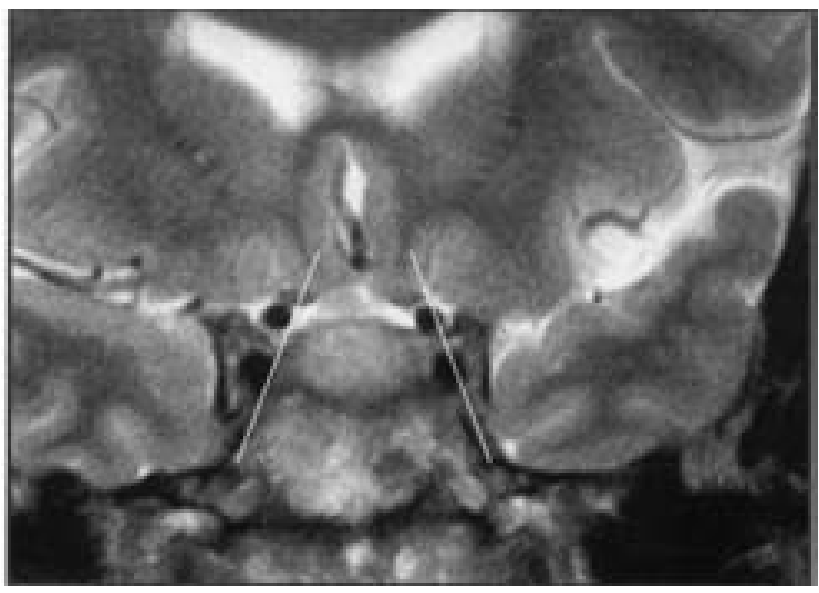

Figura 1. Derecha: El tumor no sobrepasa la tangente medial de la carótida supra-intracavernosa. (Grado 0). Izquierda: El tumor no sobrepasa la tangente medial (Grado 1)

das. El tumor no sobrepasa la línea tangencial que une la pared medial de la arteria carótida interna supracavernosa con la carótida interna intracavernosa (fig 1).

Grado 1: el tumor sobrepasa la tangente medial (definida como la tangente que une los dos bordes mediales de la carótida supra e intracavernosa) pero no sobrepasa la línea tangencial que une los dos centros de la carótida supra e intracavernosa. Los compartimientos venosos superior e inferior pueden estar obstruidos según el crecimiento de la lesión sea en sentido superior o inferior respectivamente (fig 1).

Grado 2: Se caracteriza porque el tumor se extiende por fuera de la línea intercarotídea, sin sobrepasar la tangente lateral de la carótida intra-supracavernosa (fig 2).

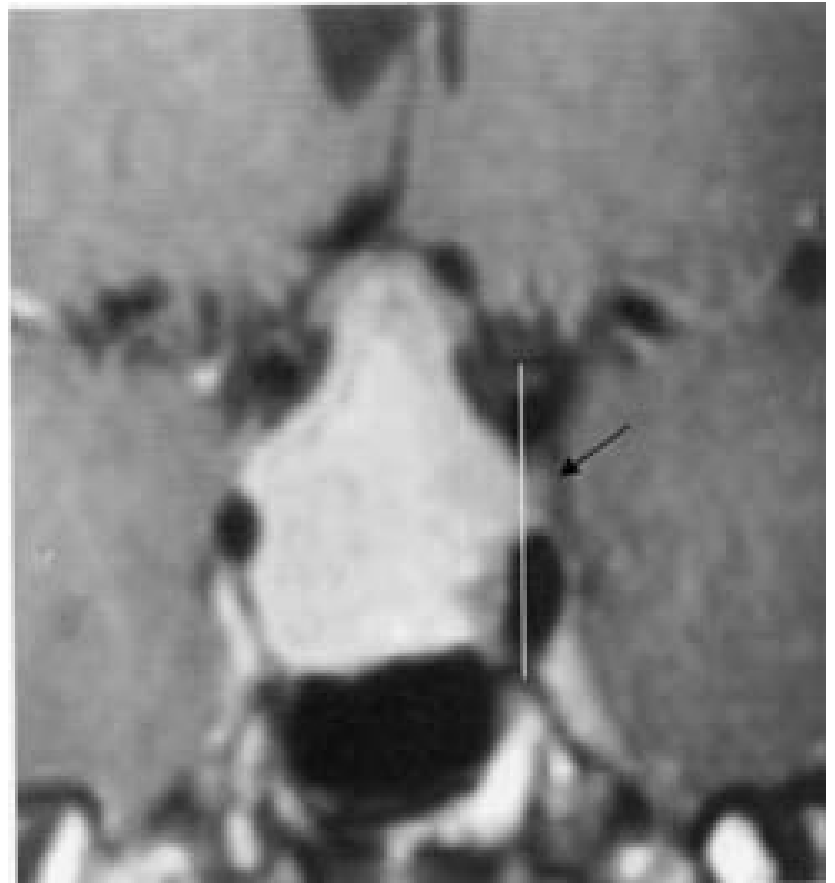

Fig 2. Tumoración que empieza a sobrepasar la tangente medial (grado 2)

Grado 3: el tumor se extiende lateralmente a la línea tangencial lateral que une la porción carotídea supracavernosa con la intracavernosa. Los compartimientos venosos medial, superior e inferior suelen estar parcial o completamente obstruidos.

Grado 4: se caracteriza porque la carótida está totalmente englobada por el tumor, estando todos los compartimientos venosos obliterados (fig 3).

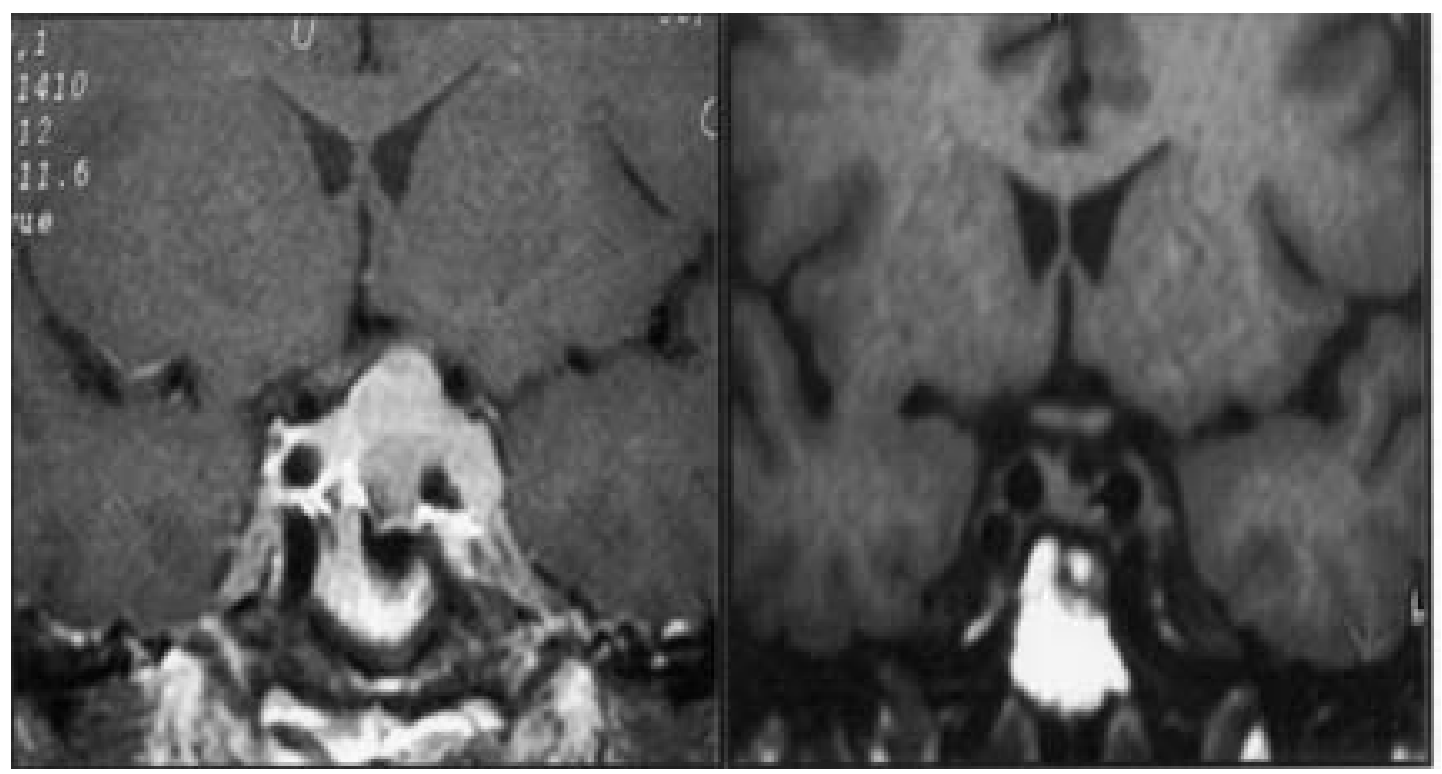

Fig 3. Tumoración grado 4 con exéresis completa. 


\section{Técnica quirúrgica}

En 20 pacientes se practicó un abordaje transesfenoidal clásico con mínimas modificaciones de la técnica quirúrgica que se resumen a continuación. Con el paciente en decúbito supino, se realiza una incisión sublabial media y una disección unilateral de la mucosa del tabique nasal. A continuación se procede a luxar el tabique nasal y se abre el rostrum esfenoidal. Una vez abierto el seno esfenoidal y resecada la mucosa, se procede a la apertura del suelo de la silla turca hasta visualizar las dos prominencias carotídeas. Se practica una apertura dural en cruz y se procede a la exéresis mediante aspiración y curetaje del tumor. Consideramos fundamental la adecuada exposición lateral de la silla turca para permitir así el curetage lateral y el acceso al interior de los senos cavernosos invadidos. Los sangrados del seno cavernoso se controlan habitualmente con facilidad mediante taponamiento con celulosa oxidada (Surgicel ${ }^{\circledR}$ Ethicon ${ }^{\circledR}$ Sarl, Suiza). Una vez terminada la exéresis del tumor se procede al relleno de la cavidad con grasa subcutánea y pegamento de fibrina (Tissucol ${ }^{\circledR}$, Baxter).

En tres pacientes de nuestra serie se realizó una exéresis transesfenoidal endoscópica de acuerdo con el procedimiento descrito por $\mathrm{Jho}^{10}$. En esencia este abordaje se practica con el paciente en decúbito supino. Previa aplicación de anestesia local tópica en la mucosa nasal, se luxa el cornete medio hasta localizar el ostium esfenoidal. A continuación, se procede a la apertura del seno esfenoidal. Una vez expuesto el suelo de la silla turca se fija el endoscopio y se procede a la apertura del mismo con una técnica similar a la expuesta anteriormente. Finalmente se realiza una comprobación del interior de la silla con los endoscopios recto y angulado a $30^{\circ}$. La fase de cierre es idéntica a la de la cirugía clásica a excepción de que en la técnica endoscópica no colocamos taponamiento en las fosas nasales.

\section{Estudio de neuroimagen y valoración endocrinológica postoperatoria}

Todos los pacientes fueron estudiados desde el punto de vista clínico, radiológico y endocrinológico (siguiendo los mismos parámetros evaluados preoperatoriamente). La primera valoración se efectuó a los tres meses del alta hospitalaria y después con una periodicidad anual. De acuerdo con los hallazgos de la RM postoperatoria se clasificaron los pacientes en las siguientes categorías: 1) resección completa, cuando no había evidencia de restos tumorales visibles en la RM de control, 2) resección subtotal cuando se consiguió una resección de más del $80 \%$ del tumor inicial y 3 ) resección parcial en el caso que la resección fuera inferior al $80 \%$ del volumen tumoral.

\section{Método estadístico}

Debido a que el estudio es fundamentalmente descriptivo se efectuó un análisis estadístico enfocado a este tipo de estudios. En todos lo casos se evaluó la distribución normal o no-normal de las variables cuantitativas mediante el método de Shapiro-Wilks. Los datos recogidos se introdujeron en una base de datos Microsoft Acces 2000 (Microsoft Corporation, Redmond, USA). Se obtuvieron tablas resumen para todas las variables recogidas en el estudio. Las variables cualitativas se resumieron mediante tablas de frecuencias con las frecuencias relativas y absolutas. En las variables continuas los estadísticos de resumen fueron la $\mathrm{N}$, la media, la desviación estándar, la mediana, el primer y tercer cuartil, y los valores mínimo y máximo. Para comparar y medir la posible asociación entre los grados de resección y de invasión se utilizó el Test Exacto de Fisher. Las decisiones estadísticas se realizaron tomando como nivel de significación el valor 0,05 . Los datos se analizaron mediante el paquete estadístico SPSS 11.0 para Windows (distribuido por SPSS inc, Chicago, IL, USA)

\section{Resultados}

Características clínicas y endocrinológicas de los pacientes

Nuestra serie consta de 15 varones y 7 mujeres, con una edad media de $46,5 \pm 12,8$ años La media de los días de estancia postoperatoria fue de 7 días y el seguimiento medio fue de 12 meses. Un paciente fue reintervenido a los tres meses por una extirpación parcial del tumor.

Clínicamente, 17 pacientes presentaban déficit visuales, 2 presentaban impotencia sexual, en 1 caso se descubrió en el estudio de una secreción purulenta nasal y en tres pacientes intervenidos anteriormente se evidenció mediante RMN de control un crecimiento de los restos postquirúrgicos. En tres pacientes la forma de presentación se caracterizó por un cuadro clínico agudo-subagudo de apoplejía hipofisaria destacando en todos ellos un déficit visual y en uno de ellos un déficit del III par craneal.

Endocrinológicamente 7 pacientes presentaban déficits FSH/LH, 7 una hiperprolactinemia, 4 hipotiroidismos, 2 hipocorticortisolismos y 1 paciente tenía una diabetes insípida.

\section{Clasificación radiológica de acuerdo con el grado de Knosp}

Radiológicamente se evaluó la invasión del seno cavernoso según la gradación de Knosp et al. ya descrita en el apartado anterior. En el caso de una afectación bilateral se valoró siempre el seno cavernoso con mayor grado de 
invasión. Cuatro pacientes se clasificaron como grado 1 , dos paciente cómo grado 2 , un paciente como grado 3 y 16 pacientes como grado 4.

\section{Resultados quirúrgicos}

Para analizar los resultados se tuvo en cuenta la última RM de control. En todos los casos (6 pacientes) con tumores grados 1 y 2 se consiguió una resección completa. En un tumor grado 3 se consiguió una resección subtotal. De los 16 pacientes con un grado 4 , en 2 se consiguió una resección completa, en 6 una resección subtotal y en 7 una resección parcial. El paciente restante falleció en el postoperatorio por una meningitis. Hemos agrupado los grupos 1 y 2 que consideramos de menor invasividad para compararlo con el conjunto de grupos 3 y 4 de una mayor invasividad y realizamos una comparación según el grado de resección:

\begin{tabular}{|c|c|c|}
\hline \multicolumn{3}{|c|}{$\begin{array}{c}\text { Tabla } 1 \\
\text { Test Exacto de Fisher }(P=\mathbf{0 . 1 2 1})\end{array}$} \\
\hline & R. total/subtotal & R. parcial \\
\hline Grados 1 y 2 & 6 & 0 \\
\hline Grados 3 y 4 & 9 & 7 \\
\hline \multicolumn{3}{|c|}{$\begin{array}{l}\text { total/subtotal (más de un } 80 \% \text { de resección) versus resec- } \\
\text { ción parcial (inferior al } 80 \% \text { ). Hemos observado que en los } \\
\text { grados } 1 \text { y } 2 \text { en todos los casos se obtuvo una resección } \\
\text { completa o subtotal, mientras que en los grados altos sólo } \\
\text { se consiguió en un } 56 \% \text { de los casos. Estas diferencias no } \\
\text { fueron estadísticamente significativas ( } \mathrm{p}=0,12 \text { ) (Tabla } 1 \text { ). } \\
\text { Los resultados quirúrgicos y anatomopatológicos se resu- } \\
\text { men en la Tabla } 2 \text {. }\end{array}$} \\
\hline
\end{tabular}

Tabla 2

Resultados quirúrgicos y anatomopatológicos

\begin{tabular}{l|l|l|l}
\hline Radiología (grados Knosp) & AP & Grado de resección & Seguimiento postoperatorio \\
\hline 4 & no secretor & subtotal & \\
4 & prolactinoma & parcial & \\
4 & no secretor & exitus & \\
4 & prolactinoma & subtotal & trat carbergolina \\
1 & fibrosis & total & \\
4 & no secretor & subtotal & \\
2 & no secretor & total & \\
4 & no secretor & parcial & \\
4 & no secretor & total & RDTEF \\
4 & no secretor & subtotal & \\
1 & necrosis & total & RDTEF \\
4 & ACTH & parcial & RDT \\
4 & fibroso, FSH & parcial & RDT \\
4 & papilar FSH & parcial & RDTEF \\
3 & no secretor & subtotal & RDTEF \\
4 & no secretor & parcial & \\
1 & ACTH & total & \\
2 & necrosis tumoral & total & RDTEF \\
1 & no secretor & total & \\
4 & no secretor & total & \\
4 & GH & subtotal & parcial \\
4 & GH & subtotal & \\
\hline
\end{tabular}

RDT: Radioterapia convencional. RDTEF: Radioterapia esterofraccionada. GH: Hormona de crecimiento. ACTH: Hormona corticotropa. FSH: Hormona foliculoestimulante 


\section{Resultados y complicaciones}

En un 94\% (16/17) de los pacientes con afectación visual se comprobó, en la campimetría postoperatoria, una mejoría de la misma, permaneciendo los déficits visuales sin cambios significativos en el paciente restante.

Los dos pacientes con disfunción eréctil por déficit de gonadotropinas no mejoraron su cuadro clínico. En los tres pacientes con apoplejía pituitaria se evidenció una recuperación visual completa así como una recuperación de la parálisis oculomotora.

Las complicaciones quirúrgicas (tabla 3 ) se dividieron en transitorias y permanentes. Las complicaciones transitorias fueron: un paciente con hiposmia, una rinitis crónica, una hipoestesia del labio superior. Un paciente presentó una parálisis completa del III par craneal que recuperó íntegramente a los 6 meses. Tres pacientes presentaron una fístula de LCR que cedieron con la colocación de un drenaje lumbar externo. De estos tres pacientes uno presentó una meningitis por E. Coli con una posterior hidrocefalia que requirió la implantación de una derivación ventrículo peritoneal. El segundo paciente falleció por una meningitis neumocócica fulminante poco después de retirar el drenaje lumbar.

A nivel endocrinológico se evidenciaron dos nuevos déficits de cortisol, un hipogonadismo, un hipotiroidismo y una diabetes insípida.

\section{Discusión}

Los adenomas de hipófisis constituyen el 13\% de los tumores intracraneales y son los tumores intraselares más frecuentes. Los recientes avances neuroquirúrgicos, junto al refinamiento en la técnica transesfenoidal y la capacidad para suplir los déficits hormonales postoperatorios, han hecho que tengan un pronóstico favorable. Aproximada- mente el 5\% de estos tumores tendrá un curso clínico más agresivo y un comportamiento biológico diferente. Este subgrupo de adenomas invasivos está asociado a una alta recurrencia con el tratamiento quirúrgico convencional.

A pesar de la gran precisión que han proporcionado los avances en las técnicas radiológicas de neuroimagen ${ }^{8,11,19}$, el diagnóstico de invasión del seno cavernoso continúa dependiendo con frecuencia de los hallazgos intraoperatorios ${ }^{7}$. Se han propuesto diferentes clasificaciones para intentar definir el grado de extensión parasellar de los macroadenomas. Aunque Hardy ${ }^{9}$ en un principio no contempló la afectación parasellar, posteriormente la introdujo como el denominado en su clasificación "estadio $\mathrm{E}^{118}$. Con posterioridad, Wilson modificó la clasificación propuesta por Hardy ${ }^{25}$ definiendo como "estadio E" la afectación de los senos cavernosos. Fahlbusch y Buchfelder ${ }^{7}$ clasificaron en 5 grados la afectación del seno cavernoso.

En la clasificación de Knosp et al. ${ }^{15}$ los grados 1 y 2 corresponden a los tipos 1 y 2 de Fahlbusch y Buchfelder ${ }^{7}$. Aunque en el tipo 3 Fahlbusch y Buchfelder (desplazamiento de todo el seno cavernoso) no tendría porqué existir invasión, Knosp et $\mathrm{a}^{15}$ concluyen que en todos los grados 3 y 4 existía una invasión del seno cavernoso confirmada quirúrgicamente. En los grados 0 y 1 de Knosp estos autores no detectaron invasión del seno mientras que fue dudosa en los grados 2 . A tenor de estos hallazgos, Knosp et al. concluyeron que el área crítica a partir de la cual podemos decir que existe una invasión del seno es cuando el tumor afecta la línea tangente lateral de la carótida supra-intracavernosa (grado 2). Nosotros hemos optado por dicha clasificación porque es de fácil manejo y distingue de forma clara y sencilla entre los diferentes grados de afectación del seno cavernoso.

Otra consideración a tener en cuenta cuando se analiza la invasividad de los macroadenomas hipofisarios es el conjunto de factores estructurales locales que puedan favo-

Tabla 3

Complicaciones postquirúrgicas

\begin{tabular}{|l|l|l|l|c|}
\hline & Transitorias & $\boldsymbol{N}$ & Definitivas & $\boldsymbol{N}$ \\
\hline \multirow{2}{*}{ Locales } & Hiposmia & 3 & Rinitis crónica & 1 \\
& Anestesia labio superior & 1 & & 1 \\
\hline \multirow{2}{*}{ Neurológicas } & Fístula LCR & 3 & Exitus & 1 \\
& Meningitis & 2 & Hidrocefalia & 1 \\
& Déficit IIIpc & 1 & Hipogonadismo & 1 \\
& & & Hipotiroidismo & 1 \\
& & & Hipocortisolismo & 2 \\
& & & Diabetes insípida & \\
\hline
\end{tabular}


recerla. Yokoyama y $\operatorname{col}^{27}$ evidencian que en 3 de sus 30 casos existían pequeños defectos a nivel de la pared medial del seno cavernoso. La propia debilidad de esta pared medial ${ }^{26}$ explicaría que en determinados casos pueda existir un desplazamiento de la pared más que una infiltración tumoral. El hecho de poder diferenciar si la pared dural del seno está invadida o únicamente desplazada podría ser un factor predictivo del grado de resección en pacientes con la carótida aparentemente englobada por el tumor. Este concepto queda remarcado por el comentario de Laws al artículo de Knosp et al. ${ }^{15}$. Lógicamente esta situación es difícilmente predecible desde el punto de vista radiológico, pero si evidenciable durante la intervención.

\section{Grado de resección quirúrgica}

El análisis de nuestros resultados objetivó una resección completa en un $36 \%$ de los pacientes. Aunque el análisis de subgrupos demuestra que en los grados 1 y 2 de Knosp ${ }^{15} \mathrm{se}$ consiguió una resección completa en todos los casos, mientras que en los grados 3 y 4 sólo en un $11 \%$. Cuando analizamos nuestros resultados, agrupando en función de los grados de resección total/subtotal $(>80 \%)$ y parcial $(<80 \%)$ y de los grados de mayor invasividad (3 y 4) y menor invasividad ( 1 y 2 ), hemos visto que las diferencias no son estadísticamente significativas $(\mathrm{p}=0,12)$; creemos que es debido al tamaño muestral de nuestra serie (error beta), aunque probablemente lo fueran si se aumentara el número de pacientes. Los resultados publicados por diferentes autores son muy variables, y algunos poco precisos en cuanto al grado de afectación del seno cavernoso. Mohr et al. ${ }^{18}$ en una serie de 77 pacientes con macroadenomas gigantes refiere una recurrencia o existencia de tumor residual en un $40 \%$. Ciric et al. ${ }^{4}$ en tumores con extensión supraselar de más de $2 \mathrm{~cm}$ observaron una tasa de recurrencias del $42 \%$ Couldwell et al. ${ }^{6}$ en una serie de 30 macroadenomas con invasión de seno cavernoso conseguían la resección completa en más de la mitad de los casos ${ }^{18}$.

Como reflexión a nuestra experiencia, pensamos que los motivos que no nos permitieron mejorar el grado de resección fueron predominantemente factores de técnica quirúrgica. El primero es que en nuestra serie no utilizamos abordajes transesfenoidales ampliados al seno cavernoso y la resección se hizo siempre a partir de un abordaje convencional. Este abordaje obliga a visualizar la pared medial del seno cavernoso en un ángulo de trabajo muy agudo que no permite una visión completa del seno cavernoso. Un segundo punto a considerar es que la utilización del material endoscópico permite una mejor visualización de estas zonas que el microscopio quirúrgico deja ocultas. En nuestra serie se incluyen sólo tres casos en que se realizó una técnica endoscópica pura, lo cual no permite extraer conclusiones sólidas de la misma. Un tercer factor a considerar son las características propias del tumor. Es lógico pensar que cuando mayor invasividad más difícil es conseguir una resección completa. Sin embargo, debemos recordar que esta es posible en algunos casos. En nuestra serie hemos conseguido resecciones completas en el grado 4. La explicación más plausible sería que, en algunos casos, la aparente invasividad del seno cavernoso no es más que un desplazamiento de la pared medial del seno sin englobamiento real de la carótida, como ya hemos explicado en el anterior apartado.

\section{Complicaciones y seguimiento}

El abordaje transesfenoidal es un procedimiento razonablemente seguro, y con una mortalidad baja en la mayoría de las series inferior al $1 \% \%^{3,5,17}$. Sin embargo, la morbilidad no es despreciable ${ }^{5,17}$. De todas las series, como estándar utilizamos el trabajo de Cirio et al. ${ }^{5}$, donde este autor recoge la experiencia de 958 neurocirujanos y analiza la frecuencia de complicaciones en función del número de intervenciones practicadas por cirujano en total. Entre las causas más frecuentes de morbilidad destacan la fístula de LCR (1,5-4.2\%), la diabetes insípida (7-20\%) y los déficits de pares craneales $(0,4-1,9 \%)^{5}$. En nuestra serie, tres pacientes presentaron una fístula de LCR de los cuales dos de ellos se complicaron con meningitis. Uno de ellos falleció y un segundo presentó una hidrocefalia postmeningitis que después requirió una derivación ventrículo-peritoneal. El fallecimiento del paciente de nuestra serie ocurrió una semana después de la intervención, tras presentar una fístula de LCR, que se complicó con una meningitis neumocócica fulminante. Sólo un paciente presentó un déficit de un III par craneal postoperatorio que recuperó completamente a los pocos meses. Un paciente presentó una diabetes insípida.

En el seguimiento de los pacientes con restos tumorales en la RM de control preferimos adoptar una actitud conservadora. En los casos de resecciones totales o subtotales realizamos controles seriados de RM y sólo cuando evidenciamos una progresión tumoral realizamos radioterapia estereofraccionada (RDTEF). En los pacientes con resecciones parciales $(<80 \%)$ realizamos RDTEF tras la intervención. Dicho manejo es parecido al que hacen otros autores ${ }^{20}$.

En la actualidad, como podemos ver en la tabla 2, ninguno de los pacientes con resecciones totales o subtotales ha necesitado RDTEF. Evidentemente el corto seguimiento de nuestros pacientes hace que tengamos que ser prudentes y valorar este dato después de un seguimiento más prolongado de nuestra serie.

\section{Conclusiones}

El manejo de los macroadenomas con invasión del seno cavernoso debe ser agresivo y dirigido a practicar una resección completa. Aunque hemos conseguido resecciones 
completas en todos los grados 1 y 2 , no hemos encontrado diferencias estadísticamente significativas entre los grados de invasividad de Knosp y de resección.

\section{Bibliografía}

1. Ahmadi, J., North, C.M., Segall, H.D., Zee, C.S., Weiss, M.H.: Cavernous sinus invasion by pituitary adenomas. AJR Am J Roentgenol 1986; 146: 257-262.

2. Alfieri, A. Jho, H.D.: Endoscopic endonasal approaches to the cavernous sinus: surgical approaches. Neurosurgery 2001; 49: 354-360.

3. Black, P.M., Zervas, N.T., Candia, G.L.: Incidence and management of complications of transsphenoidal operation for pituitary adenomas. Neurosurgery 1987; 20: 920-924.

4. Ciric, I., Mikhael, M., Stafford, T., Lawson, L., Garces, R.: Transsphenoidal microsurgery of pituitary macroadenomas with long-term follow-up results. J Neurosurg 1983; 59: 395-401.

5. Ciric, I., Ragin, A., Baumgartner, C., Pierce, D.: Complications of transsphenoidal surgery: results of a national survey, review of the literature, and personal experience. Neurosurgery 1997; 40: 225-236.

6. Couldwell, W.T., Weiss, M.H., Rabb, C., Liu, J.K., Apfelbaum, R.I., Fukushima, T.: Variations on the standard transsphenoidal approach to the sellar region, with emphasis on the extended approaches and parasellar approaches: surgical experience in 105 cases. Neurosurgery 2004; 55: 539-547.

7. Fahlbusch, R. Buchfelder, M.: Transsphenoidal surgery of parasellar pituitary adenomas. Acta Neurochir (Wien) 1998; 92: 93-99.

8. Goldstein, S.J., Lee, C., Carr, W.A., Rosenbaum, H.D., Tibbs, P.A., Walsh, J.W.: Magnetic resonance imaging of the sella turcica and parasellar region. A clinical-radiographic evaluation with computed tomography. Surg Neurol 1986; 26 : 330-337.

9. Hardy, J. Vezina, J.L.: Transsphenoidal neurosurgery of intracranial neoplasm. Adv Neurol 1976; 15: 261-273.

10. Jho, H.D.: "Endoscopic transsphenoidal surgery: experience with 50 patients". J Neurosurg 1997; 87: 44-51.

11. Kaufman, B., Kaufman, B.A., Arafah, B.M., Roessmann, U., Selman, W.R.: Large pituitary gland adenomas evaluated with magnetic resonance imaging. Neurosurgery 1987; 21: 540-546.

12. Kitz, K., Knosp, E., Koos, W.T., Korn, A.: Proliferation in pituitary adenomas: measurement by MAb K1 67. Acta Neurochir Suppl (Wien) 1991; 53: 60-64.

13. Knosp, E., Kitz, K., Perneczky, A.: Proliferation activity in pituitary adenomas: measurement by monoclonal antibody Ki-67. Neurosurgery 1989; 25: 927-930.

14. Knosp, E., Kitz, K., Steiner, E., Matula, C.: Pituitary adenomas with parasellar invasion. Acta Neurochir Suppl (Wien) 1991: 53: 65-71

15. Knosp, E., Steiner, E., Kitz, K., Matula, C.: Pituitary adenomas with invasion of the cavernous sinus space: a magnetic resonance imaging classification compared with surgical findings. Neurosurgery 1993; 33: 610-617.

16. Korbonits, M., Chahal, H.S., Kaltsas, G., Jordan, S., Urmanova, Y., Khalimova, Z., Harris, P.E., Farrell, W.E., Claret, F.X., Grossman, A.B.: Expression of phosphorylated p27(Kip1) protein and Jun activation domain-binding protein 1 in human pituitary tumors. J Clin Endocrinol Metab 2002; 87: 2635-2643.

17. Laws, E.R., Jr. Kern, E.B.: Complications of transsphenoidal surgery. Clin Neurosurg 1976; 23: 401-416.

18. Mohr, G., Hardy, J., Comtois, R., Beauregard, H.: Surgical management of giant pituitary adenomas. Can J Neurol Sci 1990; 17: 62-66.

19. Nichols, D.A., Laws, E.R., Jr., Houser, O.W., Abboud, C.F.: Comparison of magnetic resonance imaging and computed tomography in the preoperative evaluation of pituitary adenomas. Neurosurgery 1988; 22: 380-385.

20. Park, P., Chandler, W.F., Barkan, A.L., Orrego, J.J., Cowan, J.A., Griffith, K.A., Tsien, C.: The role of radiation therapy after surgical resection of nonfunctional pituitary macroadenomas. Neurosurgery 2004; 55: 100-106.

21. Scheithauer, B.W., Horvath, E., Kovacs, K., Lloyd, R.V.: Tumours of the adenohypophysis. Berlin, Springer, 2000.

22. Scheithauer, B.W., Kovacs, K.T., Laws, E.R., Jr., Randall, R.V.: Pathology of invasive pituitary tumors with special reference to functional classification. J Neurosurg 1986; 65:733-744.

23. Takino, H., Herman, V., Weiss, M., Melmed, S.: Purine-binding factor (nm23) gene expression in pituitary tumors: marker of adenoma invasiveness. J Clin Endocrinol Metab 1995; 80: 1733-1738.

24. Thapar, K., Scheithauer, B.W., Kovacs, K., Pernicone ,P.J., Laws, E.R., Jr.: p53 expression in pituitary adenomas and carcinomas: correlation with invasiveness and tumor growth fractions. Neurosurgery 1996; 38: 765-770.

25. Wilson, C.B.: A decade of pituitary microsurgery. The Herbert Olivecrona lecture. J Neurosurg 1984; 61: 814-833.

26. Yasuda, A., Campero, A., Martins, C., Rhoton, A.L., Jr., Ribas, G.C.: The medial wall of the cavernous sinus: microsurgical anatomy. Neurosurgery 2004; 55: 179-189.

27. Yokoyama, S., Hirano, H., Moroki, K., Goto, M., Imamura, S., Kuratsu, J.I.: Are nonfunctioning pituitary adenomas extending into the cavernous sinus aggressive and/or invasive? Neurosurgery 2001; 49: 857-862.

Enseñat, J.; Ortega, A.; Topcewski, T.; Vilalta, J.; Obiols, G.; Mesa, J.; Sahuquillo, J.: Valor predictivo de la clasificación de Knosp en el grado de resección quirúrgica de los macroadenomas invasivos. Estudio prospectivo de una serie de 23 casos. Neurocirugía 2006; 17: 519-526

Correspondencia postal: Joaquim Enseñat Nora. Cr. Urgell 149151 Esc 2a. Ático 1. 08036 Barcelona. 